\title{
Effects of Mycoplasma pneumoniae infection on airway neurokinin-1 receptor expression in BALB/c mice
}

\author{
H. Zhang ${ }^{1}$, B. Wei ${ }^{2}$, Y.X. Shang ${ }^{1}$, X.Y. Jiao ${ }^{1}$, L. Wang ${ }^{1}$, M.B. He ${ }^{3}$, \\ X.H. Han ${ }^{1}$ and G.Z. Wang ${ }^{3}$ \\ ${ }^{1}$ Department of Pediatrics, Shengjing Hospital of China Medical University, \\ Shenyang, China \\ ${ }^{2}$ Department of Pediatrics, Military Hospital of China, Shenyang, China \\ ${ }^{3}$ Department of Microbiology and Parasitology, China Medical University, \\ Shenyang, China
}

Corresponding author: Y.X. Shang

E-mail: yunxiaoshang@yeah.net

Genet. Mol. Res. 13 (4): 8320-8328 (2014)

Received July 29, 2013

Accepted December 4, 2013

Published October 20, 2014

DOI http://dx.doi.org/10.4238/2014.October.20.8

\begin{abstract}
The aim of this study was to establish a BALB/c mouse model of Mycoplasma pneumoniae (MP) infection and to explore the expression of neurokinin-1 receptor (NK1-R) in the trachea and lung tissue and changes in its relative content at different time points (on the 3rd, 7th, 14th, 21st, and 30th days after infection) in MP-infected $\mathrm{BALB} / \mathrm{c}$ mice. Immunohistochemistry and Western blot analysis were performed to determine NK1-R expression in the trachea and lung tissue and changes in relative content in MP-infected BALB/c mice. After MP infection, the expression of NK1-R on the surfaces of upper tracheal and bronchial epithelial cells, submucosa, and alveolar epithelial cells, as well as around the smooth muscle, was upregulated more significantly in the infection group than in the control group $(\mathrm{P}<$ 0.05); NK1-R protein expression was enhanced on the 3rd, 7th, 14th, 21 st, and 30th days after infection compared with that of the control
\end{abstract}


group $(\mathrm{P}<0.05)$. NK1-R expression in the trachea, bronchus, and lung tissue increased in MP-infected BALB/c mice, which may explain why wheezing occurs after MP infection.

Key words: Mycoplasma pneumonia; BALB/c mouse; NK1-R

\section{INTRODUCTION}

Mycoplasma pneumoniae (MP) is a common pathogen that causes atypical pneumonia and bronchitis in children, and its morbidity has gradually increased in recent years.

The relationship between MP and pediatric asthma has attracted the attention of researchers since the 1970s and has remained a focus until the present day. It is generally believed that MP closely correlates with the occurrence, development, and deterioration of pediatric asthma (Biscardi et al., 2004; Ou et al., 2008; Varshney et al., 2009; Guilbert and Denlinger, 2010). However, the relationship between MP infection and the pathogenesis of pediatric asthma remains unclear. Previous studies on asthma have indicated that expressions of neurokinin-1 receptor (NK1-R) in the airway and lung tissue are distinctly elevated in asthmatic rats and guinea pigs (Li et al., 2011; Li and Shang, 2012). MP infects airways and damages ciliated epithelial cells, resulting in exposure of non-myelinated vagal afferent fibers in the mucosa and submucosa, a release of sensory neuropeptide, and an increase of airway hyperresponsiveness. Whether NK1-R expression is enhanced after MP infection remains unclear. Therefore, we explored the expression of NK1-R in the trachea and lung tissue at different time points in MP-infected BALB/c mice.

\section{MATERIAL AND METHODS}

\section{Laboratory animals}

A total of 100 specific pathogen free (SPF) BALB/c mice, including 50 males and 50 females at 4-6-weeks old with body weight of 14-16 g, were provided by the Laboratory Animal Center of the General Hospital of Shenyang Military Region. All mice were fed a normal diet and each group contained 10 mice. This study was carried out in strict accordance with the recommendations of the Guide for the Care and Use of Laboratory Animals of the National Institutes of Health. The animal use protocol was reviewed and approved by the Institutional Animal Care and Use Committee (IACUC) of Shenjing Hospital of China Medical University.

\section{MP culture and colony assay}

The MP international standard strain, FH, was provided by the Department of Pathogenic Microorganisms in China Medical University. The main components in the fluid media were bovine brain-heart infusion medium, sterile fetal bovine serum, fresh yeast extract, glucose, penicillin, and phenol red at a $\mathrm{pH}$ of 7.6. Bacteria liquid for testing was diluted to $1 \times 10^{-1}-1 \times 10^{-12}$ using a serial dilution method; after constant temperature incubation at $37^{\circ} \mathrm{C}$ for 14 days, the highest dilution when red medium changed to yellow was used as the color change unit. Bacterium concentration was calculated as color changing units $(\mathrm{CCU}) / \mathrm{mL}$. 


\section{Establishment of MP infection model}

A total of $100 \mathrm{BALB} / \mathrm{c}$ mice, including 50 males and 50 females at 4 weeks of age, were randomized into an MP infection group (50 mice) and a control group (50 mice). Mice were kept in an SPF animal house and fed separately under standard conditions. The mice were intraperitoneally anesthetized with $0.05 \mathrm{~mL} 10 \%$ chloral hydrate; each BALB/c mouse in the infection group was intranasally vaccinated with $0.02 \mathrm{~mL} 10^{8} \mathrm{CCU} / \mathrm{mL}$ MP bacterium fluid with the mouse head tilted at $30^{\circ}-45^{\circ}$ to ensure that the MP bacterium fluid entered into the lower respiratory tract through natural breath movement. Mice in the control group were intranasally vaccinated with the same amount phosphate-buffered saline (PBS) rather than MP. Five normal mice were used as controls for each time point. Observation of daily activity and body weight was carried out after MP infection; samples were collected from infected and control mice at 4 time points on the 3rd, 7th, 14th, 21st, and 30th days after infection (Hardy et al., 2001; Martin et al., 2001).

\section{Animal grouping and sample collection}

BALB/c mice of the same species, body weight (14-16 g), and breeding conditions as well as equal numbers of male and female mice, were randomly divided into the following groups: MP infection of 3 days (MP3), 7 days (MP7), 14 days (MP14), 21 days (MP21), and 30 days (MP30). Respective studies were performed according to different time points and processing factors. For the control group, the same amount of PBS was intranasally vaccinated in mice rather than MP and the samples were collected at times corresponding to sampling of the experimental groups.

The mice were intraperitoneally anesthetized with $0.05 \mathrm{~mL} \mathrm{10 \%} \mathrm{chloral} \mathrm{hydrate} \mathrm{and}$ fixed in supine position before sample collection. The neck and chest were sterilized with conventional iodine and alcohol, and then the chest was opened with sterile equipment [soaked in $0.1 \%$ diethylpyrocarbonate (DEPC) for $24 \mathrm{~h}$, followed by autoclaved sterilization]. The entire lung was removed and weighed to calculate the lung wet weight, and then the left lung was collected and stored in $4 \%$ paraformaldehyde.

\section{NK1-R detection}

Immunohistochemistry was performed on pathological sections to detect NK1-R; the American Universal Imaging Corporation Image Analysis System (Bedford Hills; NY, USA) and MetaMorph (Molecular Devices; Sunnyvale, CA, USA) software were employed to detect the luminous intensity [integrated optical density (OD) total] to determine the relative content of NK1-R. First, 1 or 2 images were randomly selected as standards; the image analysis system was used to identify the shading percentage of red-yellow-blue as a standard when tinctorial-positive cells comprised more than 90\%. The OD of NK1-R at different sites on each section was defined as the relative content, and the mean value was calculated and presented as means $\pm \mathrm{SD}$; measurements were repeated for different points, and differences between groups were compared using one-way analysis of variance. $\mathrm{P}<0.05$ was considered to be statistically significant. NK1-R content in the lung tissue was detected using Western 
blot analysis and analyzed using the FlourChem V 2.0 gel imaging analysis software (Protein Simple; Santa Clara, CA, USA). The gray-scale value of each protein electrophoresis band was recorded and quantitatively analyzed. The protein content was calculated as the gray-scale value of the sample protein divided by the gray-scale value of $\beta$-actin in the same sample.

\section{Statistical analysis}

Statistical analysis was carried out using the SPSS 17.0 software (SPSS, Inc.; Chicago, IL, USA). Measurement data are reported as means $\pm \mathrm{SD}$; differences between groups were compared using one-way analysis of variance; $\mathrm{P}<0.05$ was considered to be statistically significant.

\section{RESULTS}

\section{NK1-R expression in the trachea of MP-infected BALB/c mice}

NK1-R mainly expressed on the surface of upper tracheal epithelial cells, submucosa, epithelial cells surrounding blood vessels, the surface of inflammatory cells, smooth muscle cells, and the surface of glandular cells (Figure 1A). The expression levels of NK1-R on the surface of airway epithelial cells and submucosa were similar to that of the control group on the 3rd (Figure 1B), 21st, and 30th (Figure 1C and D) days after MP infection, but were significantly enhanced on the 7th day (Figure 1E), after which expression declined on the 14th day (Figure 1F) after MP infection.

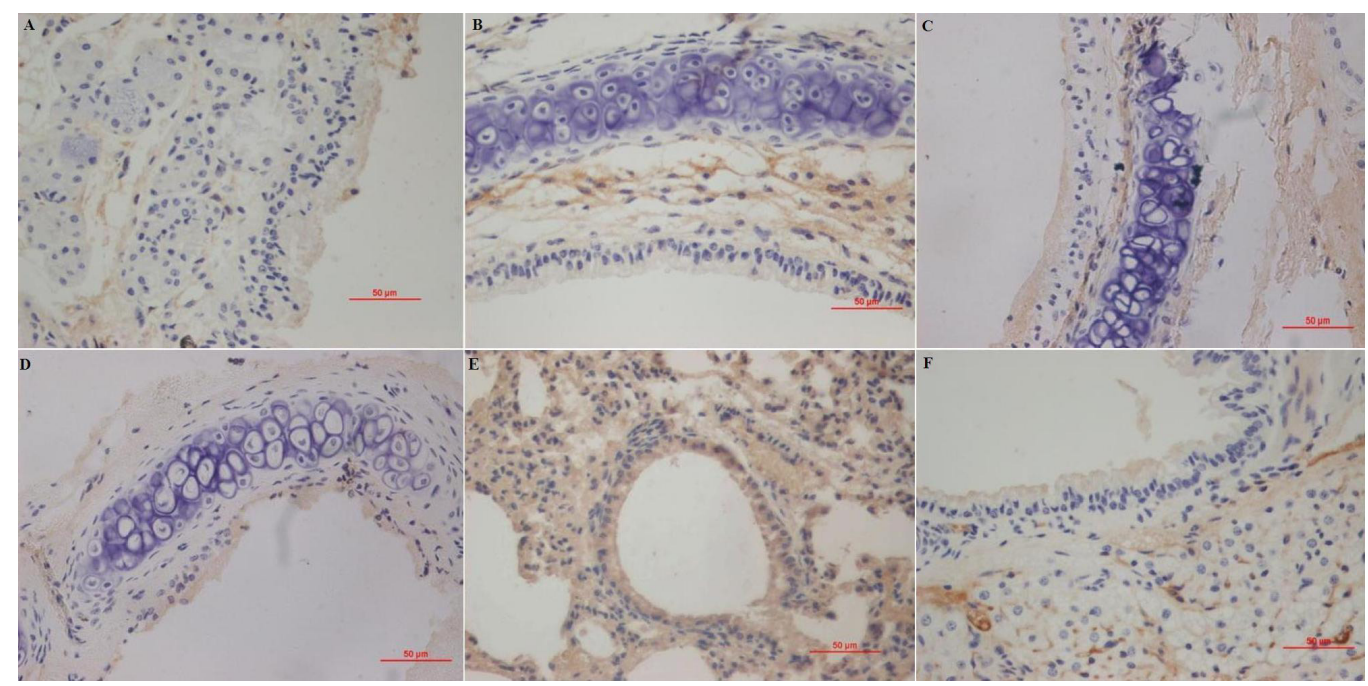

Figure 1. Expression of NK1-R protein in mouse airway from each group 400X. A. Control group; B. MP3; C. MP7; D. MP14; E. MP21; F. MP30. 


\section{NK1-R expression in the bronchus and lung tissue of MP-infected BALB/c mice}

NK1-R was predominantly expressed on the surface of upper bronchial epithelial cells, submucosa, and the surface of epithelial cells surrounding blood vessels (Figure 2A). The expression level of NK1-R on the surface of upper bronchial epithelial cells, submucosa, and alveolar epithelial cells was similar to that of the control group on the 3rd day (Figure 2B) after MP infection, but distinctly elevated on the 7th, 14th, 21st, and 30th days (Figure 2C, D, E, F) after MP infection.

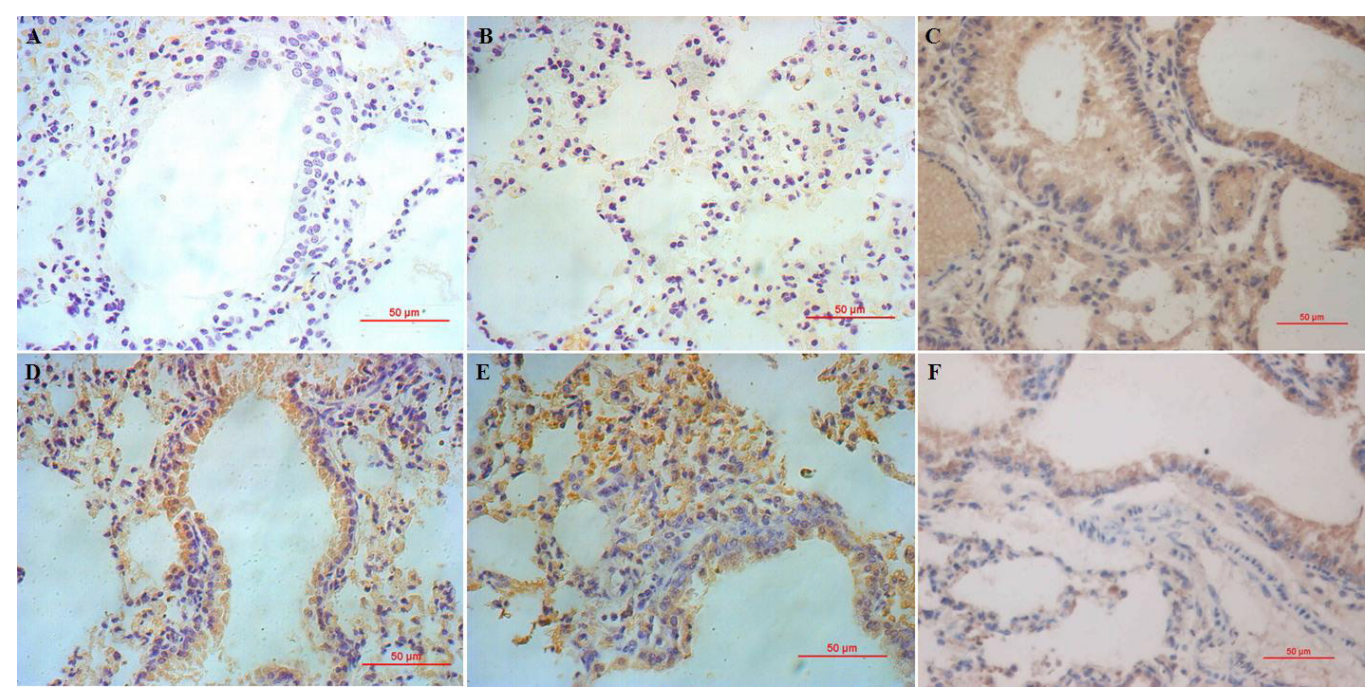

Figure 2. Expression of NK1-R protein in mouse bronchus and lung tissue from each group 400X. A. Control group; B. MP3; C. MP7; D. MP14; E. MP21; F. MP30.

\section{NK1-R expression in the bronchus and lung tissue of MP-infected BALB/c mice represented by OD}

After MP infection, NK1-R expression on the surface of upper bronchial epithelial cells, submucosa, and alveolar epithelial cells, as well as near the smooth muscle was upregulated more significantly in the infection group than in the control group $(\mathrm{P}<0.05)$, which slightly declined on the 3rd day and then increased thereafter; NK1-R expression was relatively high on the 7 th, 14 th, and 21 st days, reaching a maximum on the 7 th day and then gradually decreasing to the minimum on the 30th day after infection (Figure 3).

\section{Detection of NK1-R protein expression in mouse lung tissue from each group based on Western blot analysis}

NK1-R protein expression was enhanced on the 3rd, 7th, 14th, 21st, and 30th days after infection compared with the control group $(\mathrm{P}<0.05$, Figure $4 \mathrm{~A}$ and $\mathrm{B})$. 


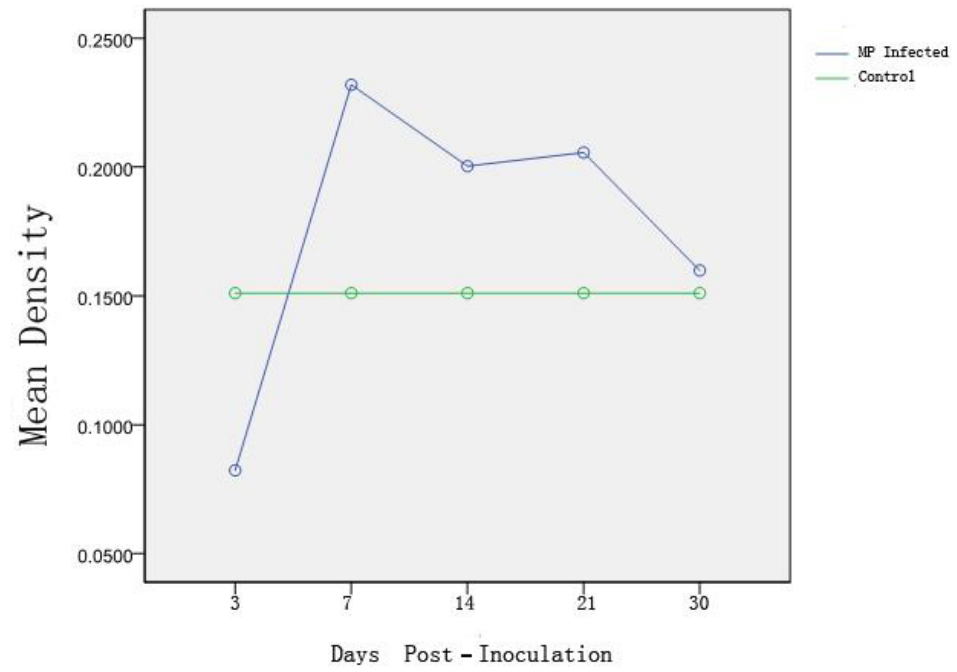

Figure 3. Relative contents of optical density reflecting NK1-R expression in bronchus and lung tissue of Mycoplasma pneumoniae-infected BALB/c mice at different time points.

A

\section{$\begin{array}{llllll}\mathrm{MP}_{3} & \mathrm{MP}_{7} & \mathrm{MP}_{14} & \mathrm{MP}_{21} & \mathrm{MP}_{30} & \mathrm{~N}\end{array}$}

nK1

actin

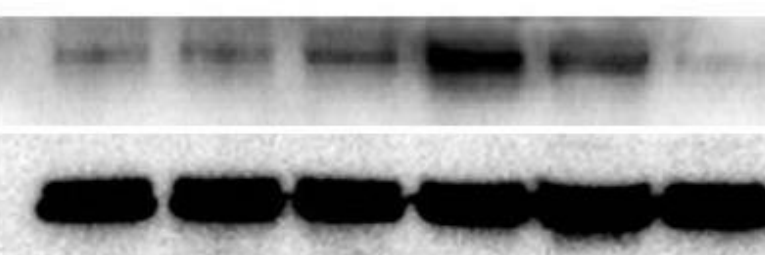

\section{B}

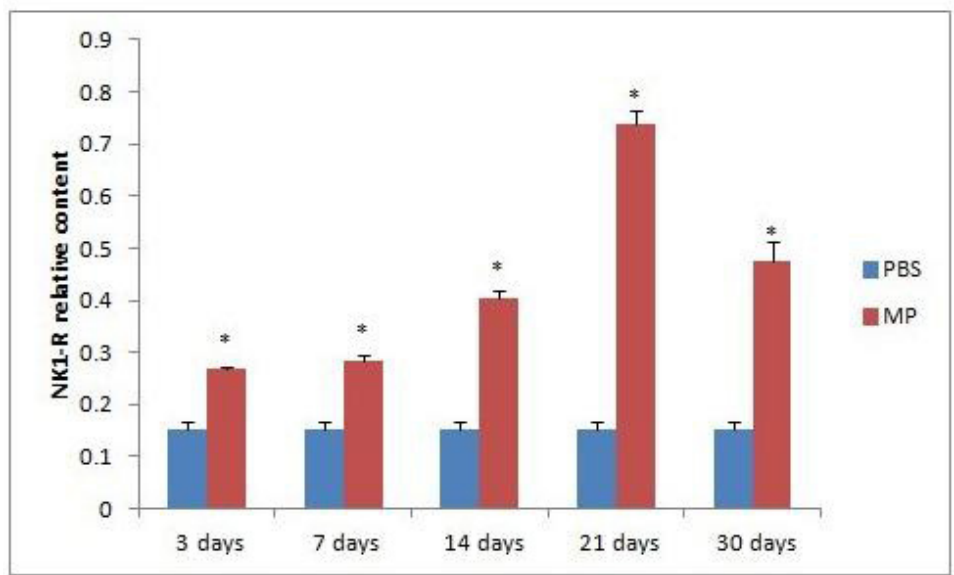

Figure 4. A. NK1-R protein expression in mouse lung tissue from each group; B. NK1-R protein expression in lung tissue of Mycoplasma pneumoniae-infected mice in each group. Lane $N=$ control group. ${ }^{*} \mathrm{P}<0.05$ was considered to be statistically significant compared with control group. 


\section{DISCUSSION}

In asthma, modulation of the NK1 receptor appears to influence a variety of pathological symptoms and processes such as inflammation, which induces pronounced and thus readily quantifiable effects in the lungs (e.g., bronchospasm, vasodilatation, vascular leakage, mucus secretion) (Groneberg et al., 2006; Satake and Kawada, 2006; Ramalho et al., 2011).

Asthma is a chronic inflammatory airway disease characterized by variable airway obstruction and bronchial hyperresponsiveness. Several factors affect the development and severity of childhood asthma such as genetic predisposition, atopy, environmental factors, obesity, diet, socioeconomic status, and infectious triggers. MP may play a role in the development of asthma exacerbation during childhood (Annagür et al., 2007).

Acute atypical bacteria are closely correlated with acute asthma attacks, and chronic MP infection plays a particularly important role in persistent asthma (Blasi and Johnston, 2007; Sutherland and Martin, 2007; Korppi, 2010; Maffey et al., 2010). An association between MP and more severe asthma exacerbation has also been observed in a previous study (Cosentini et al., 2008). However, the pathogenesis of MP in asthma remains unclear. A recent study showed that the mechanisms of wheezing attack and asthma exacerbation induced by MP infection primarily include impaired mucociliary clearance, increased mucous production, and eventually, asthma (Bisgaard et al., 2007).

In addition, MP infection stimulates multiple cells and cell components (mast cells, epithelial cells, endothelial cells, macrophages, and smooth muscle cells) to become involved in the pathophysiological process of asthma. These cells release a series of cytokines and adhesion molecules that infiltrate the airway and cause chronic airway inflammation and reconstruction (Jeong et al., 2012; Narita and Tanaka, 2012). Some researchers have hypothesized that MP sensitization and challenge induce collagen deposition in the tracheal and bronchial submucosa of BALB/c mice, resulting in airway reconstruction and causing additional severe bronchial hyperresponsiveness and asthmatic symptoms (Chu et al., 2005). Other studies (Chu et al., 2000) report that infection increases NK1-R expression in the bronchial mucosa in humans.

We observed that NK1-R expression on the surface of upper bronchial epithelial cells, submucosa, and alveolar epithelial cells, as well as near smooth muscle, slightly decreased on the 3rd day after infection and then increased thereafter; the expression level of NK1-R was relatively high on the 7 th, 14 th, and 21 st days, reaching a maximum on the 7 th day, and then gradually decreasing to a minimum on the 30th day after MP infection. In addition, NK1-R protein expression was higher on the $3 \mathrm{rd}$, 7th, 14th, 21st, and 30th days after infection compared with levels in the control group; expression of NK1-R protein increased over time to a peak on the 21 st day and gradually decreased thereafter. Both immunohistochemistry and Western blot analyses confirmed that NK1-R expression was enhanced after MP infection over time to the peak and then gradually declined. NK1-R is a crucial receptor during asthma attacks and is involved in the chronic airway inflammation induced by increased leakage of blood vessels and tracheal mucosa (Li et al., 2011). MP infection upregulates NK1-R expression in the mouse airway and lung tissue; this explains how the colonization of some pathogenic bacteria make the body more prone to wheezing (Chu et al., 2006; Bisgaard et al., 2007). Infection with MP can precede the onset of asthma, thereby exacerbating asthmatic symptoms, which makes asthma management difficult (Hong, 2012). Moreover, clarithromycin therapy reduced the concentrations of mucosal tumor necrosis factor- $\alpha$, interleukin- $1 \beta$, and interleukin-10 in children with an acute exacerbation of recurrent wheezing (Fonseca-Aten et 
al., 2006). Macrolides have both antimicrobial and anti-inflammatory properties. Both mechanisms appear to be important in their clinical efficacy in treating a wide variety of pulmonary disorders, including asthma (Good et al., 2012; Medford, 2012).

\section{REFERENCES}

Annagür A, Kendirli SG, Yilmaz M, Altintas DU, et al. (2007). Is there any relationship between asthma and asthma attack in children and atypical bacterial infections; Chlamydia pneumoniae, Mycoplasma pneumoniae and Helicobacter pylori. J. Trop. Pediatr. 53: 313-318.

Biscardi S, Lorrot M, Marc E, Moulin F, et al. (2004). Mycoplasma pneumoniae and asthma in children. Clin. Infect. Dis. 38: 1341-1346.

Bisgaard H, Hermansen MN, Buchvald F, Loland L, et al. (2007). Childhood asthma after bacterial colonization of the airway in neonates. N. Engl. J. Med. 357: 1487-1495.

Blasi F and Johnston SL (2007). The role of antibiotics in asthma. Int. J. Antimicrob. Agents 29: 485-493.

Chu HW, Kraft M, Krause JE, Rex MD, et al. (2000). Substance P and its receptor neurokinin 1 expression in asthmatic airways. J. Allergy Clin. Immunol. 106: 713-722.

Chu HW, Rino JG, Wexler RB, Campbell K, et al. (2005). Mycoplasma pneumoniae infection increases airway collagen deposition in a murine model of allergic airway inflammation. Am. J. Physiol. Lung Cell Mol. Physiol. 289: L125-L133.

Chu HW, Breed R, Rino JG, Harbeck RJ, et al. (2006). Repeated respiratory Mycoplasma pneumoniae infections in mice: effect of host genetic background. Microbes. Infect. 8: 1764-1772.

Cosentini R, Tarsia P, Canetta C, Graziadei G, et al. (2008). Severe asthma exacerbation: role of acute Chlamydophila pneumoniae and Mycoplasma pneumoniae infection. Respir. Res. 9: 48.

Fonseca-Aten M, Okada PJ, Bowlware KL, Chavez-Bueno S, et al. (2006). Effect of clarithromycin on cytokines and chemokines in children with an acute exacerbation of recurrent wheezing: a double-blind, randomized, placebocontrolled trial. Ann. Allergy Asthma Immunol. 97: 457-463.

Good JT Jr, Rollins DR and Martin RJ (2012). Macrolides in the treatment of asthma. Curr. Opin. Pulm. Med. 18: 76-84.

Groneberg DA, Harrison S, Dinh QT, Geppetti P, et al. (2006). Tachykinins in the respiratory tract. Curr. Drug Targets 7: 1005-1010.

Guilbert TW and Denlinger LC (2010). Role of infection in the development and exacerbation of asthma. Expert. Rev. Respir. Med. 4: 71-83.

Hardy RD, Jafri HS, Olsen K, Wordemann M, et al. (2001). Elevated cytokine and chemokine levels and prolonged pulmonary airflow resistance in a murine Mycoplasma pneumoniae pneumonia model: a microbiologic, histologic, immunologic, and respiratory plethysmographic profile. Infect. Immun. 69: 3869-3876.

Hong SJ (2012). The role of Mycoplasma pneumoniae infection in asthma. Allergy Asthma Immunol. Res. 4: 59-61.

Jeong YC, Yeo MS, Kim JH, Lee HB, et al. (2012). Mycoplasma pneumoniae infection affects the serum levels of vascular endothelial growth factor and interleukin-5 in atopic children. Allergy Asthma Immunol. Res. 4: 92-97.

Korppi M (2010). Bacterial infections and pediatric asthma. Immunol. Allergy Clin. North Am. 30: 565-74.

Li M and Shang YX (2012). Inhaled corticosteroids inhibit substance P receptor expression in asthmatic rat airway smooth muscle cells. BMC Pulm. Med. 12: 79.

Li M, Shang YX, Wei B and Yang YG (2011). The effect of substance P on asthmatic rat airway smooth muscle cell proliferation, migration, and cytoplasmic calcium concentration in vitro. J. Inflamm. 8: 18.

Maffey AF, Barrero PR, Venialgo C, Fernandez F, et al. (2010). Viruses and atypical bacteria associated with asthma exacerbations in hospitalized children. Pediatr. Pulmonol. 45: 619-625.

Martin RJ, Chu HW, Honour JM and Harbeck RJ (2001). Airway inflammation and bronchial hyperresponsiveness after Mycoplasma pneumoniae infection in a murine model. Am. J. Respir. Cell Mol. Biol. 24: 577-582.

Medford AR (2012). Macrolides in asthma. Chest 141: 569-570.

Narita M and Tanaka H (2012). Late increase of interleukin-18 levels in blood during Mycoplasma pneumoniae pneumonia. Cytokine 59: 18-19.

Ou CY, Tseng YF, Chiou YH, Nong BR, et al. (2008). The role of Mycoplasma pneumoniae in acute exacerbation of asthma in children. Acta Paediatr. Taiwan 49: 14-18.

Ramalho R, Soares R, Couto N and Moreira A (2011). Tachykinin receptors antagonism for asthma: a systematic review. BMC Pulm. Med. 11: 41. 
Satake H and Kawada T (2006). Overview of the primary structure, tissue-distribution, and functions of tachykinins and their receptors. Curr. Drug Targets 7: 963-974.

Sutherland ER and Martin RJ (2007). Asthma and atypical bacterial infection. Chest 132: 1962-1966.

Varshney AK, Chaudhry R, Saharan S, Kabra SK, et al. (2009). Association of Mycoplasma pneumoniae and asthma among Indian children. FEMS Immunol. Med. Microbiol. 56: 25-31. 